\section{Reply to Townsend et al.}

\author{
European Journal of Human Genetics (2014) 22, 7; \\ doi:10.1038/ejhg.2013.95; published online 15 May 2013
}

We thank Townsend et al. ${ }^{1}$ for their comments on our recently published paper. ${ }^{2}$ In their comments, they express their disagreement with the following recommendation: '...it is suggested that... clinically manageable disorders affecting the patient... must always be disclosed to the patient' '...on the other hand, the possible disclosure of other types of information.... should be discussed and agreed upon in advance with the patient during the informed consent process'. In their view, the first sentence is paternalistic.

We fully agree with Townsend et al. ${ }^{1}$ that the autonomy of the patient has to be preserved. This means that his/her decision to not know the results of a given test should be respected. We assumed that patients, after being fully informed of the usefulness and limitations of WGS testing, would make an informed decision on whether or not to undergo the test. Once the process has started, they could decide not to have the information obtained disclosed to them. The patient should inform his/her physician accordingly, and his/her decision should be respected. However, we do believe the physician should inform the patient in writing that the results are available and on file in the doctor's office, ready to be shared if he/she changes his/her mind. When we mention that '... it is suggested that... clinically manageable disorders affecting the patient... must always be disclosed to the patient...', we refer to the responsibility of the physician to inform the patient, with the final decision to be made by the latter.

Having said that, we would like to further reflect on the situation we are discussing, where two of the basic ethical principles, autonomy and justice, should be carefully considered. When we suggest that 'clinically manageable disorders affecting the patient... must always be disclosed to the patient..., we are taking into consideration that (a) WGS is a diagnostic test; (b) in this context, the use of WGS results related to a present disorder would be useful for subsequent decisions about the management of the disease; (c) the patient, in a given moment, has made a judicious decision about the option of knowing what information WGS could provide to him/her (and the attending physician); and (d) when WGS is prescribed in the National Health Service clinical setting, the chronic constraints on economic, human and material resources mean the patient should be fully aware of the responsibility that he/she assumes when deciding to undergo a WGS test. If, for instance, a patient follows the physician's recommendation that a chest X-ray will be helpful in the differential diagnosis of a presumed pneumonia, what should the physician do if the patient asks the doctor not to use the radiographic results to determine the appropriate health-care measures? Once the X-ray has been obtained, could it be ethically acceptable not to use the results obtained considering the time and economic cost incurred? This case, while showing remarkably clear-cut differences with the one we are discussing, presents a similar ethical concern. Patients should be made aware of the responsibility accompanying their decisions regarding the use of diagnostic tests that are expected to be helpful in his/her disease management; indeed, within the public health-care system, individual diagnostic decisions do not always exclusively belong to the private patient-physician relationship, but frequently have public consequences that must be carefully considered and recalled. That is why, unless a very unpredictable situation occurs, we recommend that physicians disclose the actionable WGS results related to present diseases (Groups 1 and 2A, Table 4$)^{2}$ to those patients who have decided to undergo the test once they have been engaged in a careful, open and nondirective explanation of the risks, benefits and all other relevant aspects. It should be borne in mind that in patients affected with a genetic but uncharacterized disease, the use of WGS as a diagnostic tool frequently leads to a change in the patient's management. ${ }^{3}$

For actionable findings not related to the current disease or WGS diagnostic purposes-and we understand that actionability is not restricted to therapy or care but rather encompasses prevention as well— ${ }^{4}$ a previous agreement with the patient on the types of findings to be disclosed must be discussed and agreed upon in advance during the informed consent process (Groups $3 \mathrm{~A}$ and 4 , Table 4$)^{2}$

\section{CONFLICT OF INTEREST}

The authors declare no conflict of interest.

$$
\begin{aligned}
& \text { Carmen Ayuso }{ }^{\star, 1,2} \text {, José M Millán²,3, Marta Mancheño }{ }^{4} \text { and } \\
& \text { Rafael Dal-Ré } 5 \\
& { }^{1} \text { Department of Genetics, Fundacion Jimenez } \\
& \text { Diaz (IIS-FJD), Madrid, Spain; } \\
& { }^{2} \text { CIBERER, ISCIII, Valencia, Spain; } \\
& { }^{3} \text { Department of Genetics, Hospital La Fe, Valencia, Spain; } \\
& { }^{4} \text { Department of Otorhinolaryngology, Infanta Leonor University } \\
& \text { Hospital, Madrid, Spain; } \\
& { }^{5} \text { Clinical Research Program, Pasqual Maragall Foundation, } \\
& \text { Barcelona, Spain } \\
& \text { E-mail: cayuso@fjd.es }
\end{aligned}
$$

1 Townsend A, Rousseau F, Friedman J, Adam S, Lohn Z, Birch P: Autonomy and the patient's right 'not to know' in clinical whole-genomic sequencing. Eur J Hum Genet 2014; 22: 6 .

2 Ayuso C, Millan JM, Mancheno M, Dal-Re R: Informed consent for whole-genome sequencing studies in the clinical setting. Proposed recommendations on essential content and process. Eur J Hum Genet 2013; 21: 1054-1059.

3 Dixon-Salazar TJ, Silhavy JL, Udpa N et al: Management exome sequencing can improve diagnosis and alter patient management. Sci Transl Med 2012; 4: 138ra78.

4 Berg JS, Khoury MJ, Evans JP: Deploying whole genome sequencing in clinical practice and public health: meeting the challenge one bin at a time. Genet Med 2011; 113 . 499-504. 